\title{
Position space interpretation for generalized parton distributions
}

\author{
Matthias Burkardt ${ }^{\mathrm{a} *}$ \\ a Department of Physics, New Mexico State University, Las Cruces, NM 88011, U.S.A.
}

For an unpolarized target, the generalized parton distribution $H_{q}(x, 0, t)$ is related to the distribution of partons in impact parameter space. The transverse distortion of this distribution for a transversely polarized target is described by $E_{q}(x, 0, t)$.

\section{INTRODUCTION}

Generalized parton distributions (GPDs) [1] have attracted significant interest since it has been recognized that they can not only be probed in deeply virtual Compton scattering experiments but can also be related to the orbital angular momentum carried by quarks in the nucleon [2]. However, remarkably little is still known about the physical interpretation of GPDs, and one may ask the question: suppose, about 10-15 years from now, after a combined effort from experiment, simulation and theory, we know how these functions (i.e. GPDs) look like for the nucleon. What is it, in simple physical terms, that we will have learned about the structure of the nucleon? Of course, we will have learned something about the orbital angular momentum carried by the quarks [2], but is that all there is? In these notes, I will discuss another interesting piece of information that can be extracted from GPDs, namely how partons are distributed in the transverse plane.

In nonrelativistic quantum mechanics, the physics of form factors is illucidated by transforming to the center of mass frame and by interpreting the Fourier transform of form factors as charge distributions in that frame.

GPDs [酉] are the form factors of the same operators [light cone correlators $\hat{O}_{q}\left(x, \mathbf{0}_{\perp}\right)$ ] whose forward matrix elements also yield the usual (forward) parton distribution functions (PDFs). For example, the unpolarized PDF $q(x)$ can be expressed in the form

$q(x)=\left\langle p, \lambda\left|\hat{O}_{q}\left(x, \mathbf{0}_{\perp}\right)\right| p, \lambda\right\rangle$

while the GPDs $H_{q}(x, \xi, t)$ and $E_{q}(x, \xi, t)$ are defined as

$\left\langle p^{\prime}, \lambda^{\prime}\left|\hat{O}_{q}\left(x, \mathbf{0}_{\perp}\right)\right| p, \lambda\right\rangle=\frac{1}{2 \bar{p}^{+}} \bar{u}\left(p^{\prime}, \lambda^{\prime}\right)\left(\gamma^{+} H_{q}(x, \xi, t)+i \frac{\sigma^{+\nu} \Delta_{\nu}}{2 M} E_{q}(x, \xi, t)\right) u(p, \lambda)$,

where $\Delta=p^{\prime}-p, 2 \bar{p}=p+p^{\prime}, t=\Delta^{2}, 2 \bar{p}^{+} \xi=\Delta^{+}$, and

$\hat{O}_{q}\left(x, \mathbf{b}_{\perp}\right)=\int \frac{d x^{-}}{4 \pi} \bar{q}\left(-\frac{x^{-}}{2}, \mathbf{b}_{\perp}\right) \gamma^{+} q\left(\frac{x^{-}}{2}, \mathbf{b}_{\perp}\right) e^{i x p^{+} x^{-}}$.

${ }^{*}$ This work was supported by the DOE (DE-FG03-95ER40965)

${ }^{2}$ We will suppress the scale (i.e. $Q^{2}$ ) dependence of these matrix elements for notational convenience. In the end, the $\perp$ 'resolution' will be limited by $1 / Q$. 
In the case of form factors, non-forward matrix elements provide information about how the charge (i.e. the forward matrix element) is distributed in position space. By analogy with form factors, one would therefore expect that the additional information (compared to PDFs) contained in GPDs helps to understand how the usual PDFs are distributed in position space [3]. Of course, since the operator $\hat{O}_{q}\left(x, \mathbf{0}_{\perp}\right)$ already 'filters out' quarks with a definite momentum fraction $x$, Heisenberg's uncertainty principle does not allow a simultaneous determination of the partons' longitudinal position, but determining the distributions of partons in impact parameter space is conceiveable. Making these intuitive expectation more precise (e.g. what is the 'reference point', 'are there relativistic corrections', 'how does polarization enter', 'is there a strict probability interpretation') will be the main purpose of these notes.

\section{IMPACT PARAMETER DEPENDENT PARTON DISTRIBUTIONS}

In nonrelativistic quantum mechanics, the Fourier transform of the form factor yields the charge distribution in the center of mass frame. In general, the concept of a center of mass has no analog in relativistic theories, and therefore the position space interpretation of form factors is frame dependent.

The infinite momentum frame (IMF) plays a distinguished role in the physical interpretation of regular PDFs as momentum distributions in the IMF. It is therefore natural to attempt to interpret GPDs in the IMF. This task is facilitated by the fact that there a is Galilean subgroup of transverse boosts in the IMF, whose generators are defined as

$B_{x} \equiv M^{+x}=\left(K_{x}+J_{y}\right) / \sqrt{2} \quad B_{y} \equiv M^{+y}=\left(K_{y}-J_{x}\right) / \sqrt{2}$,

where $M_{i j}=\varepsilon_{i j k} J_{k}, M_{i 0}=K_{i}$, and $M^{\mu \nu}$ is the familiar generator of Lorentz transformations. The commutation relations between $\mathbf{B}_{\perp}$ and other Poincaré generators

$$
\begin{aligned}
{\left[J_{3}, B_{k}\right] } & =i \varepsilon_{k l} B_{l} & {\left[P_{k}, B_{l}\right] } & =-i \delta_{k l} P^{+} \\
{\left[P^{-}, B_{k}\right] } & =-i P_{k} & {\left[P^{+}, B_{k}\right] } & =0
\end{aligned}
$$

where $k, l \in\{x, y\}, \varepsilon_{x y}=-\varepsilon_{y x}=1$, and $\varepsilon_{x x}=\varepsilon_{y y}=0$, are formally identical to the commutation relations among boosts/translations for a nonrelativistic system in the plane provided we make the identification [4]

$$
\begin{array}{ll}
\mathbf{P}_{\perp} \longrightarrow \text { momentum in the plane } & P^{+} \longrightarrow \text { mass } \\
L_{z} \longrightarrow \text { rotations around } z \text {-axis } & P^{-} \longrightarrow \text { Hamiltonian } \\
\mathbf{B}_{\perp} \longrightarrow \text { generator of boosts in the } \perp \text { plane. } &
\end{array}
$$

Because of this isomorphism it is possible to transfer a number of results and concepts from nonrelativistic quantum mechanics to the infinite momentum frame. For example, for an eigenstate of $P^{+}$, one can define a (transverse) center of momentum (CM)

$\mathbf{R}_{\perp} \equiv-\frac{\mathbf{B}_{\perp}}{p^{+}}=\int d x^{-} \int d^{2} \mathbf{x}_{\perp} T^{++} \mathbf{x}_{\perp}$

where $T^{\mu \nu}$ is the energy momentum tensor. Like its nonrelativistic counterpart, it satisfies $\left[J_{3}, R_{k}\right]=i \varepsilon_{k l} R_{l}$ and $\left[P_{k}, R_{l}\right]=-i \delta_{k l}$. These simple commutation relations enable us to 
form simultaneous eigenstates of $\mathbf{R}_{\perp}$ (with eigenvalue $\mathbf{0}_{\perp}$ ), $P^{+}$and $J_{3}$

$$
\left|p^{+}, \mathbf{R}_{\perp}=\mathbf{0}_{\perp}, \lambda\right\rangle \equiv \mathcal{N} \int d^{2} \mathbf{p}_{\perp}\left|p^{+}, \mathbf{p}_{\perp}, \lambda\right\rangle,
$$

where $\mathcal{N}$ is some normalization constant, and $\lambda$ corresponds to the helicity when viewed from a frame with infinite momentum. For details on how these IMF helicity states are defined, as well as for their relation to usual rest frame states, see Ref. [5].

In the following we will use the eigenstates of the $\perp$ center of momentum operator (8) to define the concept of a parton distributions in impact parameter space $f^{\text {t }}$

$q\left(x, \mathbf{b}_{\perp}\right) \equiv\left\langle p^{+}, \mathbf{R}_{\perp}=\mathbf{0}_{\perp}, \lambda\left|\hat{O}_{q}\left(x, \mathbf{b}_{\perp}\right)\right| p^{+}, \mathbf{R}_{\perp}=\mathbf{0}_{\perp}, \lambda\right\rangle$.

It is straightforward to verify that the impact parameter dependent PDFs defined above (9) are the Fourier transform of $H_{q}$ [6] (without relativistic corrections!) ?

$$
\begin{aligned}
q\left(x, \mathbf{b}_{\perp}\right) & =\frac{|\mathcal{N}|^{2}}{(2 \pi)^{2}} \int d^{2} \mathbf{p}_{\perp} \int d^{2} \mathbf{p}_{\perp}^{\prime}\left\langle p^{+}, \mathbf{0}_{\perp}, \lambda\left|\hat{O}_{q}\left(x, \mathbf{b}_{\perp}\right)\right| p^{+}, \mathbf{0}_{\perp}, \lambda\right\rangle \\
& =\frac{|\mathcal{N}|^{2}}{(2 \pi)^{2}} \int d^{2} \mathbf{p}_{\perp} \int d^{2} \mathbf{p}_{\perp}^{\prime} H_{q}\left(x,-\left(\mathbf{p}_{\perp}^{\prime}-\mathbf{p}_{\perp}\right)^{2}\right) e^{i \mathbf{b}_{\perp} \cdot\left(\mathbf{p}_{\perp}^{\prime}-\mathbf{p}_{\perp}\right)}=\int \frac{d^{2} \boldsymbol{\Delta}_{\perp}}{(2 \pi)^{2}} H_{q}\left(x,-\Delta_{\perp}^{2}\right) e^{i \mathbf{b}_{\perp} \cdot \boldsymbol{\Delta}_{\perp}}
\end{aligned}
$$

and its normalization is $\int d^{2} \mathbf{b}_{\perp} q\left(x, \mathbf{b}_{\perp}\right)=q(x)$. Furthermore, $q\left(x, \mathbf{b}_{\perp}\right)$ has a probabilistic interpretation. Denoting $\tilde{b}_{s}\left(k^{+}, \mathbf{b}_{\perp}\right)\left[\tilde{d}_{s}\left(k^{+}, \mathbf{b}_{\perp}\right)\right]$ the canonical destruction operator for a quark [antiquark] with longitudinal momentum $k^{+}$and $\perp$ position $\mathbf{b}_{\perp}$, one finds [7]

$q\left(x, \mathbf{b}_{\perp}\right)= \begin{cases}\left.\sum_{s}\left|\tilde{b}_{s}\left(x p^{+}, \mathbf{b}_{\perp}\right)\right| p^{+}, \mathbf{0}_{\perp}, \lambda\right\rangle\left.\right|^{2} \geq 0 & \text { for } \quad x>0 \\ \left.\sum_{s}\left|\tilde{d}_{s}^{\dagger}\left(x p^{+}, \mathbf{b}_{\perp}\right)\right| p^{+}, \mathbf{0}_{\perp}, \lambda\right\rangle\left.\right|^{2} \leq 0 & \text { for } \quad x<0\end{cases}$

For large $x$, one expects $q\left(x, \mathbf{b}_{\perp}\right)$ to be not only small in magnitude (since $q(x)$ is small for large $x$ ) but also very narrow (localized valence core!). In particular, the $\perp$ width should vanish as $x \rightarrow 1$, since $q\left(x, \mathbf{b}_{\perp}\right)$ is defined with the $\perp$ CM as a reference point. A parton representation for $\mathbf{R}_{\perp}$ (7) is given by $\mathbf{R}_{\perp}=\sum_{i \in q, g} x_{i} \mathbf{r}_{\mathbf{i}, \perp}$, where $x_{i}\left(\mathbf{r}_{\mathbf{i}, \perp}\right)$ is the momentum fraction ( $\perp$ position) of the $i^{t h}$ parton, and for $x=1$ the position of active quark coincides with the $\perp \mathrm{CM}$.

In order to gain some intuition for the kind of results that one might expect for impact parameter dependent PDFs, we consider a simple model

$H_{q}\left(x, 0,-\Delta_{\perp}^{2}\right)=q(x) e^{-a \Delta_{\perp}^{2}(1-x) \ln \frac{1}{x}}$.

The precise functional form in this ansatz should not be taken too seriously, and the model should only be considered a simple parameterization which is consistent with both Regge behavior at small $x$ and Drell-Yan-West duality at large $x$. A straightforward Fourier

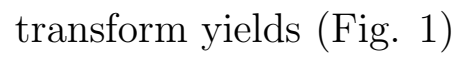

$q\left(x, \mathbf{b}_{\perp}\right)=q(x) \frac{1}{4 \pi(1-x) \ln \frac{1}{x}} e^{-\frac{\mathbf{b}_{\perp} 2}{4 a(1-x) \ln \frac{1}{x}}}$.

\footnotetext{
${ }^{3}$ Note that the Galilei invariance in the IMF is crucial for being able to construct a useful CM concept. ${ }^{4}$ In Ref. [6], wave packets were used in order to avoid states that are normalized to $\delta$ functions. The final results are unchanged. This was also verified in Ref. [8].

${ }^{5}$ A similar interpretation exists for $\tilde{H}_{q}(x, 0, t)$ in terms of impact parameter dependent polarized quark distributions $\Delta q\left(x, \mathbf{b}_{\perp}\right)=\int \frac{d^{2} \boldsymbol{\Delta}_{\perp}}{(2 \pi)^{2}} \tilde{H}_{q}\left(x, 0,-\boldsymbol{\Delta}_{\perp}^{2}\right) e^{i \boldsymbol{\Delta}_{\perp} \cdot \mathbf{b}_{\perp}}$.
} 


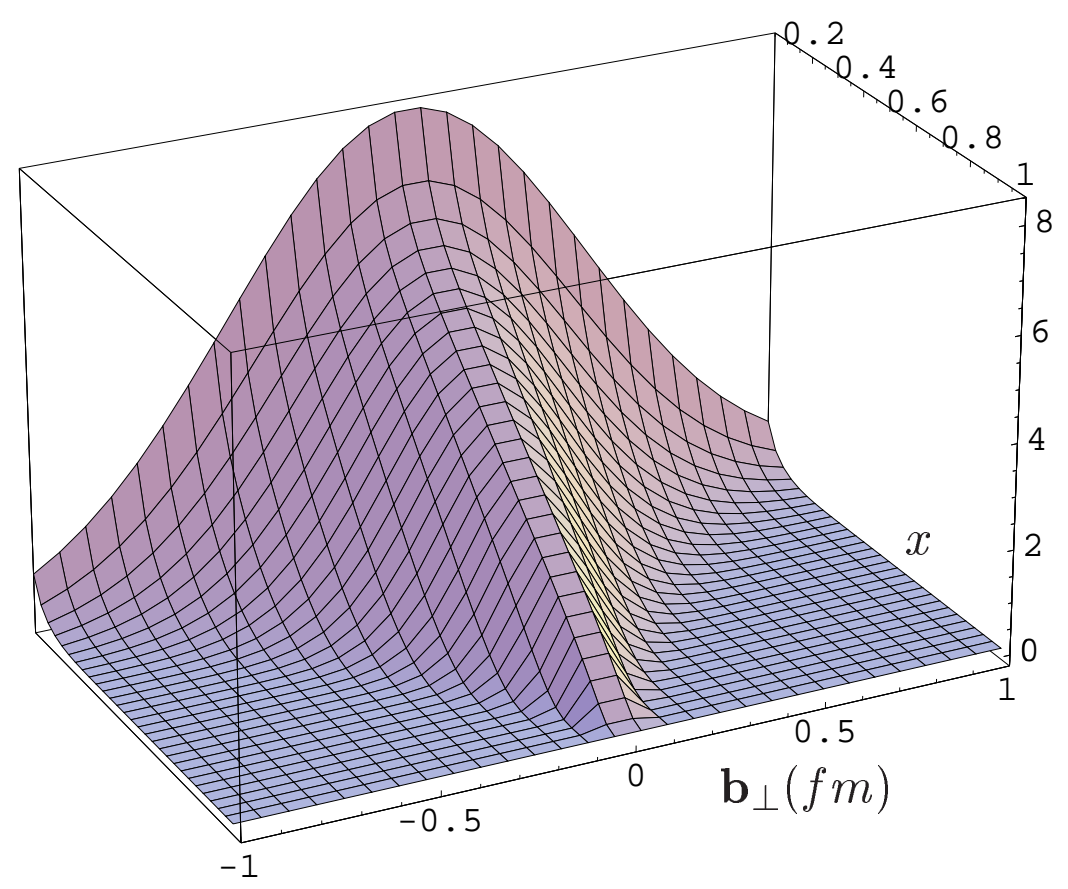

Figure 1. Impact parameter dependent parton distribution $q\left(x, \mathbf{b}_{\perp}\right)$ for the model (13).

\section{POSITION SPACE INTERPRETATION FOR $E\left(x, 0,-\Delta_{\perp}^{2}\right)$}

While both $H(x, 0, t)$ and $\tilde{H}(x, 0, t)$ are diagonal in helicity, $E(x, 0, t)$ contributes only to helicity flip matrix elements. In fact for $p^{+}=p^{+\prime}$ (i.e. $\xi=0$ ) [9] [10]

$$
\begin{aligned}
\int \frac{d x^{-}}{4 \pi} e^{i p^{+} x^{-} x}\left\langle P+\Delta, \uparrow\left|\bar{q}(0) \gamma^{+} q\left(x^{-}\right)\right| P, \uparrow\right\rangle & =H\left(x, 0,-\Delta_{\perp}^{2}\right) \\
\int \frac{d x^{-}}{4 \pi} e^{i p^{+} x^{-} x}\left\langle P+\Delta, \uparrow\left|\bar{q}(0) \gamma^{+} q\left(x^{-}\right)\right| P, \downarrow\right\rangle & =-\frac{\Delta_{x}-i \Delta_{y}}{2 M} E\left(x, 0,-\Delta_{\perp}^{2}\right)
\end{aligned}
$$

Since $E$ is off diagonal in helicity, it will therefore only have a nonzero expectation value in states that are not eigenstates of helicity, i.e. if we search for a probabilistic interpretation for $E(x, 0, t)$ we need to look for it in states that are superpositions of helicity eigenstates. For this purpose, we consider the state

$$
|X\rangle \equiv\left|p^{+}, \mathbf{R}_{\perp}=0, X\right\rangle \equiv\left(\left|p^{+}, \mathbf{R}_{\perp}=0, \uparrow\right\rangle+\left|p^{+}, \mathbf{R}_{\perp}=0, \downarrow\right\rangle\right) / \sqrt{2}
$$

In this state, we find for the (unpolarized) impact parameter dependent PDF

$$
q_{X}\left(x, \mathbf{b}_{\perp}\right) \equiv\left\langle X\left|O_{q}\left(x, \mathbf{b}_{\perp}\right)\right| X\right\rangle=\int \frac{d^{2} \boldsymbol{\Delta}_{\perp}}{(2 \pi)^{2}}\left[H_{q}\left(x, 0,-\boldsymbol{\Delta}_{\perp}^{2}\right)+\frac{i \Delta_{y}}{2 M} E_{q}\left(x, 0,-\boldsymbol{\Delta}_{\perp}^{2}\right)\right] e^{-i \mathbf{b}_{\perp} \cdot \boldsymbol{\Delta}_{\perp}}(16)
$$

Upon introducing the Fourier transform of $E_{q}$

$\mathcal{E}_{q}\left(x, \mathbf{b}_{\perp}\right) \equiv \int \frac{d^{2} \boldsymbol{\Delta}_{\perp}}{(2 \pi)^{2}} E_{q}\left(x, 0,-\Delta_{\perp}^{2}\right) e^{-i \mathbf{b}_{\perp} \cdot \boldsymbol{\Delta}_{\perp}}$ 
we thus conclude that $E_{q}$ describes how the unpolarized PDF in the $\perp$ plane gets distorted when the nucleon target is polarized in a direction other than the $z$ direction

$q_{X}\left(x, \mathbf{b}_{\perp}\right)=q\left(x, \mathbf{b}_{\perp}\right)+\frac{1}{2 M} \frac{\partial}{\partial b_{y}} \mathcal{E}_{q}\left(x, \mathbf{b}_{\perp}\right)$.

The fact that the distorted distribution is still positive implies further positivity constraints [7,11]. Above distortion (18) also shifts the $\perp \mathrm{CM}$ of the partons in the $y$-direction $\left\langle x_{q} b_{y}^{q}\right\rangle \equiv \int d x \int d^{2} \mathbf{b}_{\perp} x b_{y}^{q} q_{X}\left(x, \mathbf{b}_{\perp}\right)=-\int d x \int d^{2} \mathbf{b}_{\perp} x \frac{\mathcal{E}_{q}\left(x, \mathbf{b}_{\perp}\right)}{2 M}=-\int d x x \frac{E_{q}(x, 0,0)}{2 M}$,

i.e. the second moment of $E_{q}$ describes how far the $\perp \mathrm{CM}$ is displaced transversely in a state with $\perp$ polarization (note that the direction of the displacement is perpendicular to both the $z$ axis and the direction of the polarization. Of course, the net (summed over all quark flavors plus glue) displacement of the $\perp \mathrm{CM}$ vanishes $\sum_{i \in q, g}\left\langle x_{i} b_{y}^{i}\right\rangle=0$ [12].

The $\perp$ dipole moment due to the displacement is given by

$d_{q}^{y} \equiv \int d x \int d^{2} \mathbf{b}_{\perp} b_{y} q_{X}\left(x, \mathbf{b}_{\perp}\right)=-\int d x \int d^{2} \mathbf{b}_{\perp} \frac{\mathcal{E}_{q}\left(x, \mathbf{b}_{\perp}\right)}{2 M}=-\int d x \frac{E_{q}(x, 0,0)}{2 M}=-\frac{\kappa_{q}(0)}{2 M}$

where $e_{q} \kappa_{q}$ is the contribution from flavor $q$ to the anomalous Dirac moment $F_{2}(0)$. In order to get some feeling for the order of magnitude, we consider a very simple model where only $q=u, d$ contribute to $F_{2}(0)$, one finds for example $\kappa_{d} \approx-2$ and therefore a mean displacement of $d$ quarks of by about $0.2 \mathrm{fm}$. For $u$ quarks the effect is about half as large and in the opposite direction.

As a further illustration, we extend the model from the previous section to $E_{q}(x, 0, t)$, by making the ansatz [the factor $\frac{1}{2}$ accounts for $\int d x H_{u}(x, 0,0)=2$ ]

$E_{u}(x, 0, t)=\frac{1}{2} \kappa_{u} H_{u}(x, 0, t) \quad E_{d}(x, 0, t)=\kappa_{d} H_{d}(x, 0, t)$.

Results for $d$-quarks are shown in Fig. 22.

\section{REFERENCES}

1. X. Ji, J. Phys. G24 (1998) 1181; A.V. Radyushkin, Phys. Rev. D56 (1997) 5524; K. Goeke et al., Prog. Part. Nucl. Phys. 47 (2001) 401.

2. X. Ji, Phys. Rev. Lett. 78 (1997) 610.

3. J.P. Ralston and B. Pire, hep-ph/0110075; J.P. Ralston, B. Pire and R.V. Buniy, hep-ph/0206074.

4. J. Kogut and D.E. Soper, Phys. Rev. D 1 (1970) 2901.

5. D.E. Soper, Phys. Rev. D 5 (1972) 1956.

6. M. Burkardt, Phys. Rev. D 62 (2000) 071503.

7. M. Burkardt, proceedings of the workshop on Lepton Scattering, Hadrons and QCD, Eds. W.Melnitchouk et al., Adelaide, March 2001; hep-ph/0105324.

8. M. Diehl, hep-ph/0205208.

9. M. Diehl, Eur. Phys. J. C19, 485 (2001); P.V. Pobylitsa, hep-ph/0201030.

10. M. Diehl et al., Nucl. Phys. B 596, 33 (2001).

11. P.V. Pobylitsa, hep-ph/0204337.

12. S.J. Brodsky et al., Nucl. Phys. B 593, 311 (2001). 


$$
d\left(x, \mathbf{b}_{\perp}\right)
$$
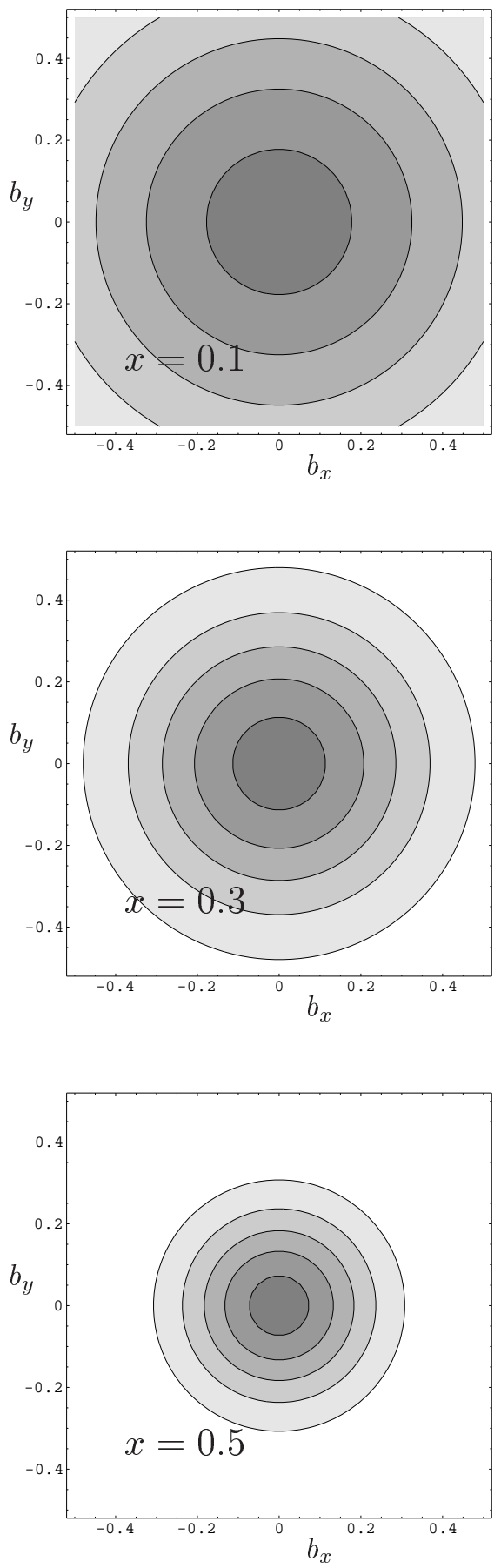

$$
d_{X}\left(x, \mathbf{b}_{\perp}\right)
$$
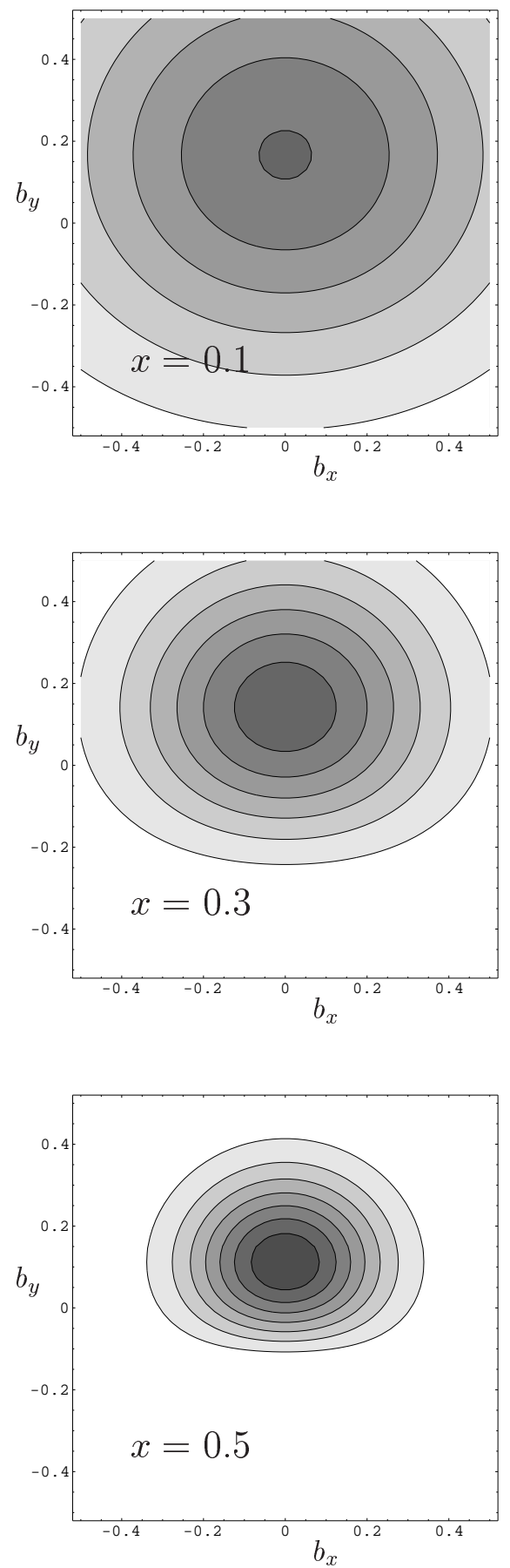

Figure 2. Impact parameter dependent PDF for $d$ quarks $d\left(x, \mathbf{b}_{\perp}\right)$ for $x=0.1,0.3,0.5$. Left column: unpolarized; right column: $d_{X}\left(x, \mathbf{b}_{\perp}\right)$ in ' $\perp$ polarized' proton . The distributions are normalized to the central value $d\left(x, \mathbf{0}_{\perp}\right)$. 